\title{
Health Care Providers' Attitudes Toward and Experiences Delivering Oral PrEP to Adolescent Girls and Young Women in Kenya, South Africa, and Zimbabwe
}

Michele Lanham ( $\nabla$ mlanham@fhi360.org )

FHI 360 https://orcid.org/0000-0003-3012-5683

Kathleen Ridgeway

FHI 360

Maryline Mireku

LVCT Health

Definate Nhamo

Pangaea Zimbabwe AIDS Trust

Diantha Pillay

Wits Reproductive Health and HIV Institute

Mercy Murire

Wits Reproductive Health and HIV Institute

Kayla Stankevitz

FHI 360

Jordan Kyongo

LVCT Health

Saiqa Mullick

Wits Reproductive Health and HIV Institute

Research article

Keywords: HIV, women, adolescent, attitude, Africa, health care providers

Posted Date: January 18th, 2021

DOl: https://doi.org/10.21203/rs.3.rs-146169/v1

License: (c) (1) This work is licensed under a Creative Commons Attribution 4.0 International License. Read Full License 


\section{Abstract}

Background: In Kenya, South Africa and Zimbabwe, oral pre-exposure prophylaxis (PrEP) is recommended for adolescent girls and young women (AGYW) at high risk of HIV. Health providers play a critical role in the uptake and effective use of sexual and reproductive health services; however, few published studies have explored providers' attitudes toward and experiences delivering PrEP to AGYW.

Methods: We conducted a cross-sectional qualitative study, interviewing 113 providers at 36 public, private, and nongovernmental health facilities in Kenya, South Africa, and Zimbabwe that were offering PrEP during the research period or were likely to offer PrEP in the future. Data were coded in NVivo 11, and an applied thematic analysis was conducted.

Results: Most providers preferred that adolescent girls wait until age 18 to have sex but acknowledged that many girls younger than 18 could benefit from oral PrEP. Their primary concern was whether adolescent girls would be able to take PrEP daily, especially if they do not tell their parents or partners they are using it. Providers reported that it was more challenging to deliver PrEP and other HIV services to girls younger than 18. Those with experience providing PrEP pointed to stigma and lack of PrEP awareness in communities as two primary barriers to PrEP uptake and use.

Conclusions: Providers were generally accepting of oral PrEP as an HIV prevention option for AGYW; however, many had negative attitudes about adolescent girls being sexually active and concerns about whether they could take PrEP daily. Results were used to update national PrEP training materials to address negative provider attitudes about PrEP use by AGYW.

\section{Background}

Adolescent girls and young women (AGYW) in eastern and southern Africa are disproportionately affected by HIV. In 2016, AGYW accounted for $26 \%$ of the regional HIV incidence, despite being only $10 \%$ of the population [1]. Existing HIV prevention options - including male and female condoms, abstinence, limiting the number of sexual partners, knowing your partner's HIV status, and antiretroviral treatment for HIV-positive partners - can be difficult for an adolescent girl or young woman to use because they require the cooperation of her male partner and prevailing gender norms hinder AGYW's decision-making power and agency in relationships [2-7].

Oral pre-exposure prophylaxis (hereafter referred to as "PrEP") has the potential to promote choice and control over HIV prevention because it can be used without a partner's knowledge or consent [8-10]. In 2015, WHO recommended daily PrEP - in the form of a tablet containing emtricitabine/tenofovir disoproxil fumarate - for use by individuals at ongoing substantial risk of HIV [11]; by August 2020, it was being offered in 68 countries [12]. In Kenya, South Africa, Zimbabwe, and other countries in southern and eastern Africa, PrEP is recommended for high-risk AGYW subpopulations, such as those who engage in transactional and intergenerational sex, experience gender-based violence (GBV), have sexually transmitted infections (STIs), are married or have multiple sexual partners, have partner(s) of unknown 
HIV status, or have older sexual partners, as well as those who perceive themselves to be at risk of HIV acquisition [13-17]. PrEP can effectively protect AGYW from HIV if they are able to access and use it as instructed [18-21].

Health providers play a critical role in the uptake and effective use of sexual and reproductive health services [22-26], and provider attitudes toward AGYW sexual activity and PrEP use are important to consider in PrEP implementation strategies [27, 28]. Studies among health providers suggest that their knowledge, attitudes, and beliefs about PrEP and potential PrEP clients influence their willingness to offer it [27, 29-31]; however, few published studies focus on providers' attitudes toward [32] and experiences with delivering PrEP to AGYW. A better understanding of providers' perceptions of PrEP use by AGYW is needed to identify potential barriers to delivery of PrEP to AGYW. In addition, the experiences of providers who are already delivering PrEP to AGYW can provide insights into barriers to PrEP uptake and use among AGYW and strategies for addressing those barriers.

The Optimizing Prevention Technology Introduction On Schedule (OPTIONS) Consortium is a USAID- and PEPFAR-funded project that provided technical assistance from 2015 to 2020 to countries rolling out oral PrEP, with a focus on AGYW. In Kenya, South Africa, and Zimbabwe, the OPTIONS Consortium provided support to national- and county-level PrEP technical working groups, which included national health officials and other key stakeholders responsible for introducing and scaling up PrEP. Among other activities, OPTIONS conducted surveys and follow-up in-depth interviews in 2017-2018 with providers in the three countries - both those experienced in PrEP delivery ("PrEP-experienced") and those who had not delivered PrEP ("PrEP-inexperienced") - to understand providers' attitudes toward and experiences with PrEP delivery to adolescent girls (ages 15 to 17) and young women (18 to 24). Conducted in the early phases of PrEP rollout at the request of the PrEP technical working groups, this research was designed to be responsive to individual country needs, ultimately informing provider training and PrEP service delivery to AGYW in each country. The provider perspectives summarized in this paper identify attitudes and experiences that could also inform PrEP rollout to AGYW in other countries in sub-Saharan Africa.

\section{Methods}

Study setting

South Africa began PrEP rollout in 2016 using a phased approach that first targeted sex workers and then expanded to include men who have sex with men (MSM) in May 2017 and AGYW in October 2017. Zimbabwe also implemented a phased approach, starting in 2015 with nongovernmental organization (NGO) clinics, and then expanding to central, district, and clinic levels, respectively; AGYW were included as a priority population for PrEP from the beginning of rollout. Kenya took a different approach, rolling out PrEP nationally starting in 2017 and targeting a number of at-risk groups, including AGYW.

Study design 
We conducted a cross-sectional qualitative study, interviewing providers at 36 public, private, and nongovernmental health facilities that were offering PrEP during the research period or were likely to offer PrEP in the future.

\section{Sampling}

Before the in-depth interviews, an initial quantitative survey was conducted with 609 providers at 60 facilities to assess providers' attitudes about PrEP for AGYW. The survey showed that some providers held attitudes indicating reservations about providing PrEP to AGYW. These results (see Additional File 1) informed the questions in in-depth interview guides developed for this study (see interview guide questions on PrEP for AGYW in Additional File 2), as well as the sampling for qualitative interviews. Survey participants were asked if they agreed to be contacted for potential participation in an in-depth interview.

Country research teams chose sites and facilities for the in-depth interviews in collaboration with the ministries of health and PrEP technical working groups to ensure the research was responsive to individual country needs. For example, we sampled a mix of sites that were and were not offering PrEP in Zimbabwe because the national Ministry of Health and Child Care (MOHCC) was preparing for PrEP scale-up at the time of the study and was interested in understanding the attitudes of both PrEPexperienced and PrEP-inexperienced providers. Kenya was already providing PrEP nationally; based on the PrEP national research agenda, $\mathrm{MOH}$ advisors wanted data from facilities providing PrEP to assess how services were being offered. Details on country-specific site and facility selection are provided in Table 1. Interviewed cadres included doctors, nurses, counselors, and community workers, selected because they have the most direct contact with PrEP clients. 
Table 1

Summary of study site and participant selection by country

\begin{tabular}{|c|c|c|c|}
\hline & Kenya & South Africa & Zimbabwe \\
\hline $\begin{array}{l}\text { Region } \\
\text { selection }\end{array}$ & $\begin{array}{l}\text { Four counties were } \\
\text { selected based on high } \\
\text { HIV incidence and } \\
\text { rural/urban mix: } \\
\text { Homabay, Kisumu, Kitui, } \\
\text { and Nairobi. }\end{array}$ & $\begin{array}{l}\text { PrEP experienced: Four } \\
\text { provinces included: Limpopo, } \\
\text { KwaZulu-Natal, Gauteng, and } \\
\text { Western Cape. Selection was } \\
\text { based on facility rather than } \\
\text { region (see below). } \\
\text { PrEP inexperienced: Three } \\
\text { sub-districts - located in the } \\
\text { provinces of Gauteng, } \\
\text { Eastern Cape, and Free State } \\
\text { - were selected from priority } \\
\text { districts for the She } \\
\text { Conquers Campaign. }\end{array}$ & $\begin{array}{l}\text { Four of the } 10 \text { provinces } \\
\text { in Zimbabwe were } \\
\text { randomly selected: } \\
\text { Harare, Manicaland, } \\
\text { Mashonaland West, and } \\
\text { Midlands. }\end{array}$ \\
\hline $\begin{array}{l}\text { Facility } \\
\text { selection }\end{array}$ & $\begin{array}{l}16 \text { facilities were selected } \\
\text { in consultation with the } \\
\text { government for the } \\
\text { survey, prioritizing } \\
\text { facilities already offering } \\
\text { PrEP. Facilities where } \\
\text { providers had the highest } \\
\text { and lowest scores in each } \\
\text { county were selected for } \\
\text { inclusion in the IDIs, } \\
\text { totaling } 13 \text { facilities. }\end{array}$ & $\begin{array}{l}\text { PrEP experienced: } 8 \text { facilities } \\
\text { providing PrEP (to sex } \\
\text { workers and MSM) and } 9 \\
\text { facilities that may provide } \\
\text { PrEP in the future (university } \\
\text { clinics, public facilities) were } \\
\text { purposefully selected for a } \\
\text { mix of delivery model (fixed } \\
\text { clinic, mobile services, or } \\
\text { both), rural/urban, and PrEP } \\
\text { uptake rate. } \\
\text { PrEP inexperienced. Five } \\
\text { facilities with sufficient } \\
\text { numbers of eligible survey } \\
\text { participants. }\end{array}$ & $\begin{array}{l}\text { Facilities providing ART } \\
\text { services to > } 50 \text { patients; } \\
26 \text { facilities selected. }\end{array}$ \\
\hline $\begin{array}{l}\text { Participant } \\
\text { selection }\end{array}$ & $\begin{array}{l}\text { Survey participants - all } \\
\text { PrEP experienced - with } \\
\text { highest and lowest } 5 \% \text { of } \\
\text { attitude scores. } \\
\text { Additional providers were } \\
\text { selected to ensure that } 10 \\
\text { providers were selected } \\
\text { from each county. }\end{array}$ & $\begin{array}{l}\text { PrEP experienced: Aimed to } \\
\text { conduct at least three IDIs } \\
\text { per facility. } \\
\text { PrEP inexperienced: Highest } \\
\text { and lowest } 15 \% \text { of attitude } \\
\text { scores and all counselors } \\
\text { and clinicians due to low } \\
\text { number of survey } \\
\text { participants from these } \\
\text { cadres. }\end{array}$ & $\begin{array}{l}\text { Survey participants with } \\
\text { highest and lowest } 10 \% \\
\text { of attitude scores. Also } \\
\text { selected all survey } \\
\text { participants currently } \\
\text { providing PrEP and three } \\
\text { additional providers who } \\
\text { did not participate in the } \\
\text { survey to ensure } \\
\text { representation of current } \\
\text { PrEP providers. }\end{array}$ \\
\hline
\end{tabular}

One author (KR) created a continuous score based on responses to the attitudinal questions in the survey described above to inform sampling for the qualitative component. Questions had five-point Likert scale response options ranging from 1, "Strongly Agree," to 5, "Strongly Disagree." Negatively framed questions, such as, "It's better to tell sexually active unmarried AGYW to abstain from sex rather than give her PrEP," were reverse-scored before creating the summary measure. The continuous score had a minimum value of 20 and maximum of 100, with higher values indicating more positive attitudes. Survey participants 
with the highest and lowest scores in each country were selected for IDI recruitment (extreme case sampling) [33]. We used this sampling approach to ensure we understood the full range of providers' attitudes about PrEP for AGYW. Additional IDI participants with high and low attitude scores were selected as needed to ensure representation of cadres, provinces/districts, facility types (public/private/nongovernmental and primary/secondary/tertiary), and sex (see Table 1).

\section{Data collection}

The research conformed to international ethical standards as stated in the United States (US) Federal Policy for the Protection of Human Subjects and was approved by the Amref Health Africa Ethics and Scientific Review Committee in Kenya, the Medical Research Council of Zimbabwe, the Wits Human Research Ethics Committee in South Africa, and the FHI 360 Protection of Human Subjects Committee in the United States. All participants provided written informed consent before participating.

Experienced male and female qualitative data collectors trained in research methods and ethics and sensitized on the study protocol conducted interviews in the participant's preferred language (English, Swahili, Kamba, or Dholuo in Kenya; English, isiXhosa, or Zulu in South Africa; and English, Shona, or Ndebele in Zimbabwe). The data collectors called survey participants who had agreed to be contacted for potential participation in an IDI and were selected using the extreme case sampling described above. Study participants did not know personal details about the data collectors and had no relationship with them prior to the study. If someone was not reached after three calls or if the survey participant refused to be interviewed, another survey participant who had agreed to be contacted for an IDI and fell within those selected by extreme case sampling was called. Interviews were scheduled with those who agreed to be interviewed. Five percent of participants who were contacted refused to participate due to various reasons, including no longer working at the target facility, refusing to be audio-recorded, being busy with other responsibilities, and being on leave. Twelve percent of participants could not be contacted.

Data collection took place from July to October 2018. Trained interviewers explained the purpose of the interviews, consented participants, and conducted in-person IDIs in private locations convenient to participants where they could not be overheard. Interviews were audio-recorded. Some participants in Zimbabwe did not consent to be audio-recorded due to concerns regarding an upcoming election. For these interviews, data collectors were accompanied by a note-taker and paused frequently to ensure the note-takers were able to document the interviews near-verbatim. Participants in Zimbabwe and Kenya were reimbursed for their time in accordance with local standards.

Data collectors used a semi-structured interview guide, available in English and local languages, that covered experiences providing PrEP and other HIV services to AGYW and attitudes about acceptable ages of sexual debut and PrEP use for adolescents. The IDIs explored attitudes separately for adolescent girls and young women. The guide also included a vignette describing a fictional adolescent PrEP client (called "Tshepiso" in South Africa, "Tafadzwa" in Zimbabwe, and "Pendo" in Kenya, but hereafter referred to as "Pendo"), with follow-up questions about the best HIV prevention options for her, whether she should disclose PrEP use to her partner and parents, concerns about her using PrEP, and what support 
she would need to use PrEP successfully. Vignettes are used in qualitative research to explore participant's attitudes and beliefs [34] .

The interview guides were pilot tested in each country. The research team met weekly during data collection to discuss the status of recruitment and data collection; the data collectors confirmed that data saturation had been reached - meaning no new themes were emerging - when the planned number of interviews had been completed [38].

Data management and analysis

The data collectors simultaneously transcribed and translated the interviews into English, and then reviewed the transcripts for accuracy. In South Africa, the transcripts were also back translated and reviewed for accuracy by an external translation company. Data were coded and analyzed by the authors - including analysts from in the US and each country - using an applied thematic analysis approach [35]. Coding was conducted in NVivo 11 [36] using a codebook developed a priori based on the interview guide, with additional thematic codes added during the analysis process. Coding was conducted by country in teams of four to six analysts, who noted key differences by PrEP-experienced versus PrEPinexperienced.

To assess inter-coder agreement, a team of eight analysts coded selected transcripts independently, compared coding, and resolved differences through discussion. Changes to the coding approach or codebook were documented, and previously coded transcripts were reviewed and updated for consistency. For each country, inter-coder agreement assessments continued until the coding team reached agreement of $80 \%$ or higher [36].

Analysts ran code reports in NVivo Version 11 and reduced and organized the data into themes [33, 35]. After synthesizing the results across the three countries, analysts compared responses to select qualitative questions by providers with positive and negative attitude scores to assess any systematic differences.

\section{Results}

Description of participants

We conducted 113 IDIs (Kenya $=40$, South Africa $=46$, and Zimbabwe $=27$ ), which lasted 55 minutes on average. Participants included 78 providers with experience delivering PrEP and 35 without PrEP experience. The average age was 33 years and the majority (70\%) were female. Experienced PrEP providers primarily provided counseling and education on PrEP. Nearly two-thirds of IDI participants were community-based workers and nurses; $91 \%$ had experience delivering health services to young women and $77 \%$ to adolescent girls (see Table 2). 
Table 2

Participant demographics

\begin{tabular}{|c|c|c|c|c|}
\hline & $\begin{array}{l}\text { Kenya } \\
(n= \\
40)\end{array}$ & $\begin{array}{l}\text { South } \\
\text { Africa } \\
(n=46)\end{array}$ & $\begin{array}{l}\text { Zimbabwe } \\
(n=27)\end{array}$ & $\begin{array}{l}\text { Overall } \\
(n= \\
113)\end{array}$ \\
\hline & $\mathrm{n}(\%)$ & $n(\%)$ & $\mathrm{n}(\%)$ & $\mathrm{n}(\%)$ \\
\hline Age, mean $\pm S D^{\star}$ & $\begin{array}{l}33.1 \pm \\
8.3\end{array}$ & $\begin{array}{l}30.0 \pm \\
10.5\end{array}$ & $38.7 \pm 9.9$ & $\begin{array}{l}33.4 \pm \\
10.1\end{array}$ \\
\hline \multicolumn{5}{|l|}{ Sex* } \\
\hline Male & $\begin{array}{l}13 \\
(33 \%)\end{array}$ & $8(21 \%)$ & $10(37 \%)$ & $\begin{array}{l}31 \\
(30 \%)\end{array}$ \\
\hline Female & $\begin{array}{l}27 \\
(68 \%)\end{array}$ & $\begin{array}{l}30 \\
(79 \%)\end{array}$ & $17(63 \%)$ & $\begin{array}{l}74 \\
(70 \%)\end{array}$ \\
\hline \multicolumn{5}{|l|}{ Position* } \\
\hline Clinician & $\begin{array}{l}11 \\
(28 \%)\end{array}$ & $2(5 \%)$ & $6(22 \%)$ & $\begin{array}{l}19 \\
(18 \%)\end{array}$ \\
\hline Nurse & $\begin{array}{l}10 \\
(25 \%)\end{array}$ & $7(18 \%)$ & $12(44 \%)$ & $\begin{array}{l}29 \\
(28 \%)\end{array}$ \\
\hline Counselor & $\begin{array}{l}9 \\
(23 \%)\end{array}$ & $6(16 \%)$ & $3(11 \%)$ & $\begin{array}{l}18 \\
(17 \%)\end{array}$ \\
\hline Community based worker & $\begin{array}{l}10 \\
(25 \%)\end{array}$ & $\begin{array}{l}23 \\
(61 \%)\end{array}$ & $6(22 \%)$ & $\begin{array}{l}39 \\
(37 \%)\end{array}$ \\
\hline \multicolumn{5}{|c|}{$\begin{array}{l}\text { Self-reported responsibilities related to PrEP (multiple } \\
\text { responses possible) }{ }^{\star \star}\end{array}$} \\
\hline Prescribing & $\begin{array}{l}17 \\
(43 \%)\end{array}$ & $3(8 \%)$ & $7(29 \%)$ & $\begin{array}{l}27 \\
(26 \%)\end{array}$ \\
\hline Resupplying & $\begin{array}{l}16 \\
(40 \%)\end{array}$ & $3(8 \%)$ & $8(33 \%)$ & $\begin{array}{l}27 \\
(26 \%)\end{array}$ \\
\hline Counseling & $\begin{array}{l}27 \\
(68 \%)\end{array}$ & $2(5 \%)$ & $13(54 \%)$ & $\begin{array}{l}42 \\
(41 \%)\end{array}$ \\
\hline Community Outreach & $\begin{array}{l}16 \\
(40 \%)\end{array}$ & $4(11 \%)$ & $8(33 \%)$ & $\begin{array}{l}28 \\
(27 \%)\end{array}$ \\
\hline Education & $\begin{array}{l}30 \\
(75 \%)\end{array}$ & $7(18 \%)$ & $16(67 \%)$ & $\begin{array}{l}53 \\
(52 \%)\end{array}$ \\
\hline None (PrEP inexperienced) & $1(3 \%)$ & $\begin{array}{l}28 \\
(74 \%)\end{array}$ & $6(25 \%)$ & $\begin{array}{l}35 \\
(34 \%)\end{array}$ \\
\hline
\end{tabular}

NOTE: some percentages add to more than $100 \%$ due to rounding; $* 8$ missing in South Africa; $* * 8$ missing in South Africa, 3 missing in Zimbabwe 


\begin{tabular}{|c|c|c|c|c|}
\hline & $\begin{array}{l}\text { Kenya } \\
(n= \\
40)\end{array}$ & $\begin{array}{l}\text { South } \\
\text { Africa } \\
(n=46)\end{array}$ & $\begin{array}{l}\text { Zimbabwe } \\
(n=27)\end{array}$ & $\begin{array}{l}\text { Overall } \\
(n= \\
113)\end{array}$ \\
\hline & n (\%) & n (\%) & $\mathrm{n}(\%)$ & $\mathrm{n}(\%)$ \\
\hline \multicolumn{5}{|l|}{$\begin{array}{l}\text { Self-reported service delivery experience with (Multiple } \\
\text { responses possible)** }\end{array}$} \\
\hline Adolescent girls & $\begin{array}{l}34 \\
(85 \%)\end{array}$ & $\begin{array}{l}22 \\
(58 \%)\end{array}$ & $23(96 \%)$ & $\begin{array}{l}79 \\
(77 \%)\end{array}$ \\
\hline Young women & $\begin{array}{l}35 \\
(88 \%)\end{array}$ & $\begin{array}{l}34 \\
(89 \%)\end{array}$ & $24(100 \%)$ & $\begin{array}{l}93 \\
(91 \%)\end{array}$ \\
\hline Self-reported familiarity with PrEP for HIV prevention* & $\begin{array}{l}38 \\
(95 \%)\end{array}$ & $\begin{array}{l}21 \\
(55 \%)\end{array}$ & $23(85 \%)$ & $\begin{array}{l}82 \\
(78 \%)\end{array}$ \\
\hline 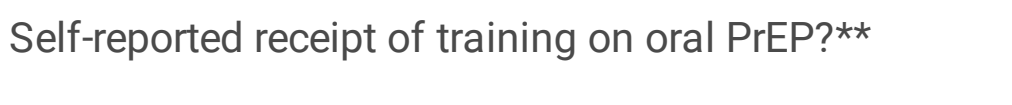 & $\begin{array}{l}17 \\
(43 \%)\end{array}$ & $8(21 \%)$ & $8(33 \%)$ & $\begin{array}{l}33 \\
(32 \%)\end{array}$ \\
\hline
\end{tabular}

Attitudes toward and perceptions of PrEP for AGYW

Results are summarized by interview topic below, and illustrative quotes are presented in Table 3. The results were similar across countries unless otherwise noted. Across these topics, one-third of providers who participated in IDIs voiced one or more concerns or hesitations, but few expressed consistently negative attitudes or serious concerns throughout the interview. For example, a PrEP-experienced clinician in Kenya stated that PrEP was a good option for "Pendo," the 17-year-old girl in the vignette, because, "she has fears that the boyfriend has several sexual partners," but the clinician recommended that she first try to abstain from sex because "she is an adolescent. That means she can wait to get to the mature age." When asked about offering Pendo PrEP, the clinician replied, "For now no, because you know these drugs are meant for preventive measures and they have to be used as prescribed. So, if there is any nonadherence issue, she is at risk of getting HIV." However, the clinician also stated that they would be comfortable offering PrEP to Pendo if she were someone they knew: "I am not concerned about what the lady is using, my concern is about what she might get... we just want to prevent [...] HIV." 
Table 3

Illustrative quotes

Acceptable age for sexual activity and PrEP use
They are still young...this is when they are still in high school ... where you concentrate. It shapes your life from there, so sex can wait. - Nurse, Kenya, previously provided PrEP

It's inappropriate for adolescents between age 15 and 18 to engage in sexual intercourse...they shouldn't take PrEP either. But if they feel [...] they are wise enough to engage in such thing, then it's okay. [...] It's their choice. I mean they have rights but with these rights come responsibilities. - Community worker, South Africa, not providing PrEP

As a mother, if I see a girl of 17 years taking PrEP, I wouldn't like it. But that is the reality of what's happening with these girls today. As a health worker I have seen PrEP helping others, so it's rather better for her to take the PrEP.

- Nurse, Zimbabwe, providing PrEP

Best HIV prevention options

For me, I would go for PrEP because like I said, women have low bargaining power. [...] You know she cannot tell the boyfriend to use a condom. - Clinician, Kenya, not providing PrEP

She should use both oral PrEP and at the same time using protection [condoms] [...] At least she will have a backup of oral PrEP. Sometimes maybe they can use condoms, [but] at the same time the guy might refuse to use condoms. At least at that time she will be using oral PrEP.

- Nurse, Zimbabwe, not providing PrEP

Concerns about PrEP

She won't be able to take it correctly because she's afraid of being found out by [her partner]. [...] Maybe he could react negatively and beat her or ask why she didn't tell him that she is using this prevention.

- Community worker, South Africa, not providing PrEP

She will stop [using condoms]. [...] She will say "I'm protected. I can't get HIV" [...] she knows she is protected she can't get HIV so there are some risky behaviors [...] I will [still offer her PrEP] because she is willing to take them [PrEP] home. I think everybody knows his or herself. I know she will find a way of how to take them despite the husband knowing.

- Counselor, Kenya, providing PrEP

Male partners
I usually leave [disclosure] at her discretion, if she wants to its okay and if she does not want to, it's still okay [...] it's at her comfort because she is the one who knows the boyfriend. [...] I would just tell her first try and ask him if he is willing to go for HIV test. If you see the reaction is bad, that means if you start telling him about the PrEP it will also bring more issues to it, so she would rather keep it to herself.

- Nurse, Kenya, providing PrEP

[Her partner] is not the person to talk to [...] he will react negatively and also he may even get violent because now at the moment she strongly suspects him of having multiple partners. If she says that she is taking PrEP because of that, maybe they may get into a fight, so I don't think that she should tell him. - Community worker, South Africa, not providing PrEP 
Parents Yes, at her age she should tell her parents that she is using oral PrEP. She must inform them so that she is able to take them consistently, and she can also experience side effects and they will not know how best to help her.

\section{- Community worker, Zimbabwe, not providing PrEP}

\section{At times someone can come and pick PrEP but later call to inform me, "my mother threw away the drugs. Maybe she doesn't understand what kind of drugs they are." - Community educator, Kenya, providing PrEP}

Support needed
Whoever will be administering [PrEP] to her just to check on her least once a month or so [to see] if she takes her pills regularly and how she is doing, if she has side effects or anything. I think she needs an emotional support.

- Community worker, South Africa, not providing PrEP

[She will need] A lot of counseling and advice. And in that particular moment before she starts, I will even try to engage the partner... to talk on behalf of her on the importance of taking this [PrEP] and why it is not a crime on taking them...

- Clinician, Kenya, providing PrEP

[Pendo] needs adherence counseling and side effects management, appointment reminders and relationship counseling would be necessary too [...]l would also tell her that PrEP does not protect her from pregnancy and STIs.

- Counselor, Zimbabwe, not providing PrEP

Service delivery to AG versus YW

Uptake, adherence, retention challenges
[Women ages] 20-24 years, I have seen that their [PrEP] retention rate is a little better in comparison to those below 20. Yes, I think they more focused, they know their problems, they know their risk and they are determined.

- Nurse, Zimbabwe, providing PrEP

At least at that age 20-24 [years] someone has started to mature even when you give them information [...] they show up, they listen. You know at around 20 years, someone already knows what they want to do with their lives so they can't let you down.

- Community worker, Kenya, providing PrEP

They [AG] are afraid of their parents, they are afraid of the community, they are afraid of going to access these services because they feel that they are judged by the providers [...] with that fear comes the denial that they are sexually active.

-22-year-old female community educator, South Africa, does not provide PrEP

They are worried about being caught taking [PrEP] tablets cause it's not known in the community. Their boyfriends do not know about it, so if they see their tablets they will think that they are positive. - Clinician, Zimbabwe, providing PrEP

The challenge is just about stigma [...] If an adolescent girl comes who is HIV-negative and wants PrEP ...the next question is how would my parents take me because the drugs are stored at home, so once the parent sees the bottle maybe the parent can have a notion that this girl maybe she is behaving [way] that is not pleasing to the parent ... to the society she is taking PrEP because her behavior is not good. 
Strategies used to address challenges
There are times when I go beyond and give the adolescents my WhatsApp phone number so that they may get in touch if they have a problem. It is because they do not have anyone to talk to. It becomes easier for them to be in touch if they have a problem after we create rapport. We can be in contact by phone before things escalate. - Nurse, Zimbabwe, not providing PrEP

I think creating awareness and letting them know that it's actually okay to come to those clinics and actually talking to not only them but the communities to know that because HIV is not only an adolescents' issue but a community issue. To make people aware that this is just another way to prevent this disease from spreading even wider.

- Community educator, South Africa, not providing PrEP

We exchange phone numbers so we can talk. When we realize they have missed appointments I say, 'You were supposed to come today.' So I make a follow up using WhatsApp, and if I can't get hold of her I ask the peer counselors to make a follow up.

- Nurse, Zimbabwe, providing PrEP

\section{Acceptable age for sexual activity and PrEP use}

Many providers said girls engaged in sexual activity at an earlier age than they thought was appropriate. The majority preferred that girls wait until they are 18. The reasons providers cited for this preference included that 18 is the age when girls typically complete secondary school, the legal age of consent in Zimbabwe and Kenya, and the age when young people become "more mature." A few - mostly PrEPinexperienced providers - felt strongly that ages 15 to 19 were "too young" for sex and that "sex could wait."

Despite their concerns about adolescent girls being sexually active, IDI participants acknowledged that many adolescent girls need HIV prevention education and those at risk of HIV could benefit from PrEP. Most IDI participants said that it is acceptable for AGYW to initiate PrEP when they are 15 to 19 years old or as soon as they are sexually active. Only a small minority of providers said 15 to 19 was "too young" to initiate PrEP, because they are "children" and should "study first."

\section{Best HIV prevention options}

When providers were asked what they thought were the best HIV prevention options for Pendo, condoms and PrEP were mentioned most frequently, though few talked about using them simultaneously. They discussed the relative advantages and disadvantages of these methods, acknowledging that neither is perfect. They noted that condoms have the advantage of preventing HIV, other STIs, and pregnancy, while PrEP prevents only HIV. Condoms were also viewed as easier for young people to access and use.

Providers said the main advantage of PrEP for Pendo was that she could use it without the knowledge or consent of her partner, in contrast to condoms, which would likely be challenging for her to negotiate. However, many acknowledged that PrEP use might be difficult to conceal from a partner, who might react negatively to its use. When asked which method they would recommend Pendo try first, most said condoms, because they provide protection against other STIs and pregnancy as well as HIV. In Zimbabwe, some responses varied by PrEP experience. A few providers, most of whom were PrEP- 
experienced, recommended that she try PrEP in conjunction with condoms, while the majority, who were largely PrEP-inexperienced, recommended she try condoms alone.

\section{Concerns about PrEP}

Participants' primary concern with Pendo using PrEP was whether she would be able to adhere to daily use, especially if her partner or parents disapproved or if she was trying to use it without their knowledge. A small minority of providers were concerned about the potential for decreased condom use and increased risky behavior. They reasoned that once Pendo was on PrEP, "there is nothing she will be worried about." They also pointed out that her partner might pressure her not to use condoms.

Despite their concerns, most providers said they would offer PrEP to Pendo because of her risk of HIV and potential difficulty negotiating the use of other HIV prevention methods. They said they "want her to be safe," and that it is better to take PrEP than "leaving things to chance." A few providers - mostly PrEPinexperienced - were opposed to offering Pendo PrEP, primarily because of concerns about her ability to adhere to daily use.

Participants were asked if their responses would change if Pendo were someone they knew. Most maintained an accepting attitude, saying they would have "no problem" or would be "very happy," because it would be better for her to use PrEP than to be at risk of HIV. Some said they would offer support and encouragement to help Pendo use PrEP effectively. Others had concerns about her being sexually active at "such an early age" and acknowledged the disconnect between how they felt as health providers and how they felt as parents.

\section{Male partners}

Participants said that the decision of whether to disclose PrEP use to a male partner was complex. Those who thought Pendo should disclose PrEP use to her partner said disclosure could facilitate adherence and continuation. She would not have to worry about hiding her PrEP use, and her partner could remind her to take PrEP daily or help her get refills. Others stated that disclosure was important to ensuring trust and transparency in her relationship. Many providers, especially in Kenya, stated that disclosure might encourage her partner to get tested or start using PrEP himself.

Several participants thought Pendo's decision should be based on how much negotiating power she has in the relationship and how she thinks her partner will react, particularly whether he might react violently. Participants reasoned he might misunderstand her PrEP use to indicate that she does not trust him, has other partners, or is HIV-positive. Some providers in South Africa viewed PrEP use as an individual decision more than a relational one, calling it "a personal thing" that her partner "doesn't have to know about," and saying that "it's her life." Providers recommended that Pendo gauge her partner's potential reaction by bringing up HIV testing and prevention generally, or that she use partner and/or couples' HIV counseling and testing services to facilitate disclosure of PrEP use.

\section{Parents}


Participants were asked about Pendo disclosing PrEP use to parents. One-third to one-half of participants in each country said Pendo should disclose to her parents because they could provide encouragement and could support her adhering to daily use, obtaining PrEP refills, and managing side effects. Those who thought she should not disclose to her parents said there was "no need" or thought her parents would not react well. Disclosure would reveal that Pendo is sexually active, which her parents might not approve of; some even said that telling her parents would "destroy the relationship." They also said parents may not understand what PrEP is because of low community awareness about it, including mistaking PrEP for antiretroviral treatment (ART). Even providers who thought Pendo should disclose noted that disclosure could be challenging for these same reasons.

Some providers said Pendo should decide whether to disclose based on the nature of her relationship with her parents. For example, if her parents were "modern," "open-minded," or "understanding," she could talk to them about her PrEP use, but if they were "strict" it would be better not to discuss PrEP with them. A few providers in Zimbabwe had specific recommendations, such as suggesting that Pendo talk to her mother more generally about PrEP first, to gauge her reaction, or involve a counselor in the discussion.

\section{Differences between participants with positive and negative attitudinal scores}

In the IDIs, providers with positive attitudes from the survey tended to show more accepting attitudes regarding initiating PrEP at younger ages, and those with negative attitude scores more often reported that they thought adolescent girls should wait until they are 18 or older to have sex. When asked in IDIs what HIV prevention option they would recommend for Pendo, providers with negative attitudes from the survey most often recommended that she try condoms and abstinence or end the relationship. Providers with positive attitudes from the survey more often expressed that PrEP was a good option for Pendo. There were no differences in provider concerns about PrEP by provider attitude score, demonstrating that while providers may have had overall very high or very low attitudinal scores, both had a variety of concerns about PrEP for AGYW. Similarly, provider responses did not differ by attitude scores when asked what they would advise if Pendo was someone they knew and providers in both groups expressed similar opinions about disclosure to and support from Pendo's partner and parents.

\section{Experiences delivering PrEP and other HIV services to AGYW \\ Service delivery to AG versus YW}

Reflecting on their experiences providing PrEP and other HIV services, providers across the three countries reported that they faced more challenges delivering services to adolescent girls compared with young women. They characterised adolescent girls as "troublesome," "not listening," "reluctant" to seek services or receive information, "clueless" about their partners' behavior and "dishonest." By comparison, they described young women as "more mature," "focused," willing to talk about sex and to "listen more." and knowing how to "take care of themselves." 
These perceptions were attributed to the difference in life stages. Providers noted that young women live on their own or are married with children, which gives them more freedom to seek services and increased motivation to continue taking PrEP, whereas adolescents are more likely to live at home and be in school, which poses challenges of parental involvement and reduced mobility and access to services. Providers also said age differences can make it challenging for them to relate to adolescent girls. They suggested that adolescent girls may feel more comfortable discussing sensitive topics with peers or younger health workers.

\section{Uptake, adherence, and continuation challenges}

IDI participants with experience providing PrEP confirmed that uptake, adherence, and continuation among AGYW have been challenging, particularly for adolescent girls. They cited lack of PrEP awareness, stigma, and lack of PrEP disclosure to partners and parents as the primary barriers. Some also mentioned side effects as a barrier to PrEP use.

PrEP-experienced providers said PrEP awareness is low among AGYW and communities; most notable is the lack of understanding about the differences among PrEP, post-exposure prophylaxis (PEP), and ART. They also talked about the difficulties AGYW experienced using PrEP without telling their partners and parents. Lack of awareness about PrEP in the community and fear of negative reactions were cited as the primary reasons for not disclosing PrEP use to partners and parents. Providers reported that some parents received specific information about PrEP that helped garner their support and were then able to facilitate their daughters' PrEP adherence.

Providers talked about two types of stigma as barriers to PrEP uptake and use: HIV stigma and stigma related to being sexually active. They said AGYW are concerned that community members who see a young woman access PrEP service will assume she is taking antiretroviral pills because she is HIVpositive. They also worry about parents or partners discovering the tablets or hearing them rattle in the bottle. Providers explained that adolescents feared being stigmatized if they used PrEP or accessed other HIV services, because that would mark them as someone who is sexually active, and they would be labeled "promiscuous" or assumed to be engaged in sex work. Providers said AGYW clients did not report experiencing stigma related to PrEP use, but their fear of being stigmatized was an important influence on their service-seeking behavior. Providers discussed HIV stigma as an issue that affected adolescent girls more than young women.

\section{Service delivery strategies}

PrEP providers shared strategies they were using to facilitate PrEP uptake, adherence, and continuation, including intensified counseling to address adherence and continuation challenges; community discussions to address stigma and normalize PrEP for AGYW; and peer support groups to encourage adolescent girls to share their challenges. They also track missed client visits and follow up with calls, SMS, and home visits to promote continuation. Providers discussed strategies used in other HIV services that could be applied to PrEP, including using social media and digital platforms to reach AGYW; supporting AGYW-parent relationships through counseling, assisted disclosure, and health talks with 
parents; encouraging couples' HIV testing and counseling; structuring services to improve anonymity and confidentiality; and providing services at places and times convenient for students.

\section{Discussion}

Providers were generally supportive of PrEP for AGYW, but they had more reservations about PrEP for adolescent girls compared to young women. Hesitations about providing PrEP to adolescent girls were primarily related to: (1) negative attitudes about adolescent girls being sexually active and (2) concern about the ability of AG to adhere to daily use, particularly when using PrEP without disclosing to parents and partners. These concerns are consistent with studies of provider attitudes toward AGYW PrEP use $[27,32]$, and they are supported by data from trials and national programs, which show that younger age is associated with lower adherence and continuation [41, 42]. Other studies have shown that providers' concerns about behavioral disinhibition could negatively affect their willingness to provide PrEP $[43,44]$. While some providers in this study had concerns about behavioral disinhibition in the form of decreased condom use and increased risky behavior among PrEP users, this did not emerge as a major concern.

Participants with experience providing PrEP reported that stigma, lack of PrEP awareness among AGYW and communities, and fear of disclosing PrEP use to partners and parents were the main barriers to PrEP uptake and use among AGYW. These findings are consistent with barriers identified by young women, community members, and health care providers in other studies [41, 44-50]. In our research, most providers pointed to stigma from community members rather than providers as a barrier, but it is possible that participants did not recognize their own stigmatizing attitudes or did not want to discuss them. Other studies have found that fear of being stigmatized is a deterrent to discussing PrEP with a provider [44, 47, 51] and that some providers in sub-Saharan Africa limit provision of sexual and reproductive health services to adolescents due to negative attitudes about them being sexually active [52].

Our findings suggest the need for additional sensitization of providers and communities - including stigma reduction - to promote PrEP as an HIV prevention method for AGYW. Even among study participants who demonstrated primarily positive attitudes about PrEP for AGYW, about one-third shared a concern or negative attitude about a particular aspect of delivering PrEP to AGYW.

A study in Tanzania found that better provider training was associated with more willingness to provide PrEP to AGYW [43]. Training that includes values clarification and delivering adolescent-friendly services $[43,53,54]$ may be the highest priorities for provider training. Values clarification training helps providers reflect on their personal views about adolescent girls being sexually active and the attitudes underlying their concerns about behavioral disinhibition to become more aware of how their personal views affect how they provide services [55]. Values clarification should also address attitudes about PrEP for AGYW, because some providers expressed negative attitudes about PrEP for this population and providers may have implicit biases they are unaware of [56]. Providers need training and job aids to counsel AGYW on whether and how to talk to their male partners and parents about PrEP use [57]. 
Many providers in this study expressed concerns about the ability of AGYW to adhere to PrEP and use it successfully; however, lessons learned from ART programs suggest that PrEP should still be offered to AGYW despite potential or even likely adherence challenges [58]. Promising approaches to improving PrEP adherence need to be evaluated with AGYW. These include peer support, weekly communication from providers, more frequent clinic visits, integration of PrEP delivery into comprehensive sexual health programs, text messaging and PrEP apps, relationship counseling, cash incentives, and adherence clubs $[41,46,49,57,59,60]$.

Despite the concerns and challenges that providers in this study identified about delivering PrEP to adolescent girls, most acknowledged that adolescent girls could benefit from PrEP because of their risk of HIV and inability to negotiate condoms. Further, most said they would be willing to offer PrEP to adolescent girls. Providers' stated recognition of AG's need for additional HIV prevention methods and their willingness to provide it are encouraging. These findings are consistent with another study of providers' attitudes about PrEP for AGYW in Tanzania [27].

Our results suggest that intensive community sensitization to promote PrEP awareness and combat stigma $[41,45,47,61]$ is crucial to making PrEP a viable HIV prevention option for AGYW and other vulnerable populations. Specific messages and events for parents, male partners, and community leaders could promote awareness, provide accurate information about PrEP, and help AGYW who want to use PrEP gain the support of their parents and partners. At the same time, building the skills and efficacy of stigmatized groups to challenge stigma has been identified as a best practice [62]; training AGYW to be PrEP ambassadors in their communities could help reduce PrEP stigma and promote AGYW PrEP use [63, 64]. Finally, promoting PrEP as a method for anyone who wants to promote wellness, rather than a method for high-risk - and often highly stigmatized - groups, could help reduce stigma [49, 65-67].

Findings from this study have influenced provider training in the study countries. OPTIONS developed an AGYW Provider Training Package that included values clarification exercises to address specific negative attitudes and beliefs revealed in this study [68]. These values clarification exercises were incorporated by the MOHCC in Zimbabwe into national PrEP provider trainings. In Kenya, the National PrEP Curriculum was updated to include the vignette used in this study, and questions about provider attitudes toward PrEP for AGYW were shared with the National AIDS and STI Control Programme, counties, and PrEP implementing partners for use or adaptation to assess PrEP providers' attitudes. In South Africa, study findings informed the revision of the national PrEP service provider training to address emerging concerns regarding PrEP provision to adolescent girls.

Study results also informed the development of an HIV Prevention Ambassador Training Curriculum for Adolescent Girls and Young Women, which prepares AGYW ambassadors to support their peers to learn about PrEP as part of combination HIV prevention and to support peers who decide to use PrEP. To address the barriers to AGYW PrEP use that providers raised in this study, we included tools in the curriculum to: 1) help AGYW develop skills to talk with their parents and partners about PrEP and 2) address misconceptions about PrEP, including the difference between PrEP, PEP, and ART. OPTIONS has 
used the curriculum to train more than 175 ambassadors in Kenya, South Africa, and Zimbabwe. Trained ambassadors have supported PrEP community awareness campaigns in Chitungwiza, Zimbabwe, and Kiambu County, Kenya.

\section{Limitations}

Providers' attitudes about PrEP for AGYW were self-reported and may not be entirely accurate given the likelihood of implicit bias. Barriers to PrEP uptake, adherence, and continuation were reported from the point of view of providers and may not fully reflect clients' experiences. In addition, data collection occurred when the three countries were in the early stages of national PrEP rollout; it is possible provider perspectives have evolved with experience and as additional provider training and community awareness campaigns have been conducted.

Another limitation of this study is that the samples differ by country, because the sampling approach for each country was designed to be directly responsive to in-country needs and priorities and was not designed for forming cross-country comparisons. During country-specific analyses, similar themes emerged across countries, leading to the decision to report the multi-country findings. Although we noted results that emerged from a specific country, we were unable to systematically compare results across countries because of the difference in samples by country. Nonetheless, the convergent results point to important issues that could be addressed to improve PrEP service delivery for AGYW. These results could be used to inform future research that could investigate differences in providers' attitudes based on provider age, PrEP experience, cadre, or gender or explore to what extent provider attitudes were shaped by country-level differences in culture, policy, or PrEP rollout strategy.

\section{Conclusions}

In summary, providers who participated in this research were generally accepting of PrEP as an HIV prevention option for adolescent girls and young women; however, many had negative attitudes about adolescent girls being sexually active. They also had concerns about whether adolescent girls could take PrEP daily, particularly given the lack of PrEP awareness in the community and the challenges of disclosing PrEP use to parents and partners. To promote effective delivery of PrEP for AGYW, national PrEP provider training in Kenya, South Africa, and Zimbabwe was updated to address negative attitudes about adolescent sexual activity and concerns about PrEP. In addition, a training curriculum for AGYW HIV prevention ambassadors was created to address potential barriers to PrEP use identified in this study. These findings could inform the introduction and scale-up of PrEP for AGYW in other countries and potential future introduction of new ARV-based HIV prevention methods such as the dapivirine ring and injectable PrEP.

\section{Declarations}

\section{Ethics approval and consent to participate}


The study was approved by the Amref Health Africa Ethics and Scientific Review Committee in Kenya, the Medical Research Council of Zimbabwe, the Wits Human Research Ethics Committee in South Africa, and the FHI 360 Protection of Human Subjects Committee in the United States.

\section{Consent for publication}

Not applicable

\section{Availability of data and materials}

The datasets generated and/or analyzed during the current study are not publicly available due to potential sensitivity of the content but are available from the corresponding author on reasonable request.

\section{Competing interests}

The authors declare that they have no competing interests.

\section{Funding}

\section{Authors' contributions}

$\mathrm{ML}, \mathrm{KR}, \mathrm{MM}, \mathrm{DN}, \mathrm{DP}, \mathrm{MMu}, \mathrm{KS}$, JK, and SM conceptualized and designed the studies. MM, DF, DP, MMu, and $\mathrm{JK}$ collected the data. $\mathrm{ML}, \mathrm{KR}, \mathrm{MM}, \mathrm{DN}$, and $\mathrm{MMu}$ analyzed the qualitative data. $\mathrm{ML}, \mathrm{MM}$, and $\mathrm{KR}$ wrote the paper. DN, DP, MMu, KS, JK, and SM reviewed and edited the manuscript. All authors have read and approved the final version.

\section{Acknowledgements}

OPTIONS Consortium Research Team (in alphabetical order): Taurai Bhatasara, Lina Digolo, Theresa Hoke, Annrita Ikahu, Patriciah Jeckonia, Jordan Kyongo, Michele Lanham, Megan Lydon, Nicole Makahamadze, Maryline Mireku, Wanjiru Mukoma, Saiqa Mullick, Mercy Murire, Joseph Murungu, Getrude Ncube, Definite Nhamo, Christian Ochieng, Diantha Pillay, Subarna Pradhan, Kathleen Ridgeway, Katie Schwartz, Patience Shamu, and Kayla Stankevitz

The authors would like to thank the study participants and data collectors. We would also like to acknowledge the South Africa National Department of Health, the Zimbabwe Ministry of Health and Child Care, the Kenya National AIDS and STI Control Programme (NASCOP), and the county health management teams in Nairobi, Kisumu, Homabay, and Kitui counties for their support of this research.

\section{References}

1. UNAIDS. Ending. AIDS: progress towards the 90-90-90 targets. Geneva: UNAIDS; 2017. 
2. Elias CJ, Coggins C. Female-controlled methods to prevent sexual transmission of HIV. AIDS. 1997;10(Suppl 3):43-51.

3. Raphael MC. Microbicides are promoted as offering a 'female-controlled' HIV prevention method: so can they revolutionize the HIV crisis of young women in Kenya? J Public Health. 2012;34(4):625-30.

4. Saul J, Moore J, Murphy ST, Miller LC. Relationship violence and women's reactions to male- and female-controlled HIV prevention methods. AIDS Behav. 2004;8(1090-7165): 207 - 14.

5. Marcus R, Page E. Changing discriminatory norms affecting adolescent girls through communication activities: a review of evidence. London: Overseas Development Institute; 2014.

https://www.odi.org/publications/8538-changing-discriminatory-norms-affecting-adolescent-girlsthrough-communication-activities-review. Accessed 21 Sep 2020.

6. Muralidharan A, Fehringer J, Pappa S, Rottach E, Das M, Mandal M. Transforming gender norms, roles, and power dynamics for better health: evidence from a systematic review of gender-integrated health programs in low- and middle-income countries. Washington (DC): Futures Group, Health Policy Project; 2015. https://www.measureevaluation.org/resources/publications/tr-15-118. Accessed 21 Sep 2020.

7. World Health Organization (WHO). Promoting gender equality to prevent violence against women. Geneva: WHO; 2009. https://www.who.int/violence_injury_prevention/violence/gender.pdf. Accessed 21 Sep 2020.

8. Braksmajer A, Senn TE, McMahon J. The potential of pre-exposure prophylaxis for women in violent relationships. AIDS Patient Care STDS. 2016;30(6):274-81.

9. Heffron R, Pintye J, Matthews LT, Weber S, Mugo N. PrEP as peri-conception HIV prevention for women and men. Curr HIV/AIDS Rep. 2016;13(3):131-9.

10. Aaron E, Blum C, Seidman D, Hoyt MJ, Simone J, Sullivan M, et al. Optimizing Delivery of HIV Preexposure Prophylaxis for Women in the United States. AIDS Patient Care STDS. 2018;32(1):1623.

11. World Health Organization (WHO). Guideline on when to start antiretroviral therapy and on preexposure prophylaxis for HIV. Geneva: WHO; 2018.

12. AVAC. prepwatch.org [Internet]. New York: AVAC; 2020. https://www.prepwatch.org/. Accessed 21 Sep 2020.

13. Birdthistle I, Schaffnit SB, Kwaro D, Shahmanesh M, Ziraba A, Kabiru CW, et al. Evaluating the impact of the DREAMS partnership to reduce HIV incidence among adolescent girls and young women in four settings: a study protocol. BMC Public Health. 2018;18(1):912.

14. Dunbar MS, Kripke K, Haberer J, Castor D, Dala S, Mukoma W, et al. Understanding and measuring uptake and coverage of oral pre-exposure prophylaxis delivery among adolescent girls and young women in sub-Saharan Africa. Sexual Health. 2018;15(6):513-21.

15. Celum CL, Delany-Moretlwe S, McConnell M, van Rooyen H, Bekker LG, Kurth A, et al. Rethinking HIV prevention to prepare for oral PrEP implementation for young African women. J Int AIDS Soc. 2015;18(4Suppl 3):20227. 
16. The Global Fund. The Global Fund measurement framework for adolescent girls and young women programs. Geneva: The Global Fund; 2018.

17. Pilgrim N, Mathur S, Gottert A, Rutenberg N, Pulerwitz J. Building evidence to guide PrEP introduction for adolescent girls and young women. Washington (DC): Population Council; 2016.

18. ATHENA Initiative. A review of the pre-exposure prophylaxis (PrEP) research and evidence landscape in Kenya and Uganda for adolescent girls and young women. Washington (DC): JSI Research \& Training Institute; 2017.

19. The Global Fund. Adolescent girls and young women in high-HIV burden settings. Geneva: The Global Fund; 2017.

20. Kirby T. Targeting HIV prevention to young women in Africa. The Lancet. 2016;388(10060):2579.

21. Pulerwitz J. HIV Prevention in the hands of adolescent girls and young women: acceptability and feasibility of oral PrEP in Tanzania. New York: The Population Council; 2018.

22. Glinski A, Sexton M, Petroni S. Understanding the adolescent family planning evidence base. Washington [DC]: International Center for Research on Women; 2014.

23. Singh NS, Aryansinghe SA, Smith J, Khosla R, Say L, Blanchet K. A long way to go: a systematic review to assess the utilisation of sexual and reproductive health services during humanitarian crises. BMJ Global Health. 2018;3(2):000682.

24. Bolton L, Bradford C. Adolescent sexual and reproductive health. Oxford: HEART; 2016.

25. Dassah CW, Cornelius AT, Edward T. Determinants of contraceptive use and future contraceptive intentions of women attending child welfare clinics in urban Ghana. BMC Public Health. 2017;18(1):79.

26. FHI 360. Examining the influence of providers on contraceptive uptake in Rwanda. Durham (NC): FHI $360 ; 2013$.

27. Pilgrim N, Jani N, Mathur S, et al. Provider perspectives on PrEP for adolescent girls and young women in Tanzania: the role of provider biases and quality of care. PLoS One. 2018;13(4):132018.

28. Pleuhs B, Quinn KG, Walsh JL, Petroll AE, John SA. Health care provider barriers to HIV pre-exposure prophylaxis in the United States: a systematic review. AIDS Patient Care STDs. 2020;34(3):111-23.

29. Castel AD, Feaster DJ, Tang W, Willis S, Jordan H, Villamizar K, et al. Understanding HIV care provider attitudes regarding intentions to prescribe PrEP. J Acquir Immune Defic Syndr. 2015;70(5):520-8.

30. Mathur S, Pilgrim N, Pulerwitz J. PrEP introduction for adolescent girls and young women. Lancet HIV. 2016;3(9):e406-e8.

31. Ross I, Mejia C, Melendez J, Chan PA, Nunn AC, Powderly W, et al. Awareness and attitudes of preexposure prophylaxis for HIV prevention among physicians in Guatemala: implications for countrywide implementation. PloS One. 2017;12(3):e0173057.

32. Mullins TLK, Idoine CR, Zimet GD, Kahn JA. Primary care physician attitudes and intentions toward the use of HIV pre-exposure prophylaxis in adolescents in one metropolitan region. J Adolescent Health. 2019;64(5):581 - 8.33. 
33. Tolley EE, Ulin PR, Mack N, Robinson ET, Succop SM. Qualitative methods in public health: a field guide for applied research. 2nd ed. San Francisco: Jossey-Bass Public Health; 2016.

34. Finch J. The vignette technique in survey research. Sociology. 1987;21(1):105-14.

35. Soydan $\mathrm{H}$. Using the vignette method in cross-cultural comparisons. Cross-national research methods in the social sciences. 1996:120-8.

36. Hughes R, Huby M. The construction and interpretation of vignettes in social research. Social Work Social Sciences Review. 2012;11(1):36-51.

37. Guest G, MacQueen KM, Namey EE. Applied thematic analysis. Thousand Oaks (CA): Sage Publications, Inc.; 2011.

38. QSR International. Ltd QIP. NVivo qualitative data analysis software. Version 11. 2018. https://www.qsinternational.com/nvivo/home.

39. Holsti OR. Content analysis for the social sciences and humanities. Reading (MA): Addison-Wesley; 1969.

40. Guest G, Bunce A, Johnson L. How many interviews are enough? An experiment with data saturation and variability. Field Methods. 2006;18(1):59-82.

41. Buchbinder SP, Liu AY. CROI 2019: advances in HIV prevention and plans to end the epidemic. Top Antivir Med. 2019;27(1):8.

42. Marrazzo JM, Ramjee G, Richardson BA, Gomez K, Mgodi N, Nair G, et al. Tenofovir-based preexposure prophylaxis for HIV infection among African women. N Engl J Med. 2015;372(6):50918.

43. Pilgrim N, Jani N, Mathur S, Kahabuka C, Saria V, Makyao N, et al. Provider perspectives on PrEP for adolescent girls and young women in Tanzania: the role of provider biases and quality of care. PloS One. 2018;13(4):e0196280.

44. Calabrese SK, Underhill K. How stigma surrounding the use of HIV preexposure prophylaxis undermines prevention and pleasure: a call to destigmatize "Truvada whores". Am Journal Public Health. 2015;105(10):1960-4.

45. Kambutse I, Igiraneza G, Ogbuagu O. Perceptions of HIV transmission and pre-exposure prophylaxis among health care workers and community members in Rwanda. PloS One. 2018;13(11):e0207650.

46. Sidebottom D, Ekström AM, Strömdahl S. A systematic review of adherence to oral pre-exposure prophylaxis for HIV-how can we improve uptake and adherence? BMC Infect Dis. 2018;18(1):581.

47. Maxwell S, Gafos M, Shahmanesh M. Pre-exposure prophylaxis use and medication adherence among men who have sex with men: a systematic review of the literature. J Assoc Nurses AIDS Care. 2019;30(4):e38-61.

48. Bradley E, Forsberg K, Betts JE, DeLuca JB, Kamitami E, Porter SE, et al. Factors affecting preexposure prophylaxis implementation for women in the United States: a systematic review. J Women's Health (Lrchmt). 2019;28(9):1272-85. 
49. Celum CL, Delany-Moretlwe S, Baeten JM, van der Straten A, Hosek S, Bukusi EA, et al. HIV preexposure prophylaxis for adolescent girls and young women in Africa: from efficacy trials to delivery. J Int AIDS Soc. 2019;22(Suppl 4):e25298.

50. Digolo L, Ochieng C, Ngunjiri A, Kiragu M, Kyongo J, Otiso L, et al. Uptake of and retention on HIV preexposure prophylaxis (PrEP) among adolescent girls and young women in Kenya. 8th International Workshop on HIV \& Women; The Colonnade Hotel, Boston: LVCT Health; 2018.

51. Brooks RA, Landrian A, Nieto O, Fehrenbacher A. Experiences of anticipated and enacted preexposure prophylaxis (PrEP) stigma among Latino MSM in Los Angeles. AIDS Behav. 2019;23(7):1964-73.

52. Jonas K, Crutzen R, van den Borne B, Reddy P. Healthcare workers' behaviors and personal determinants associated with providing adequate sexual and reproductive healthcare services in subSaharan Africa: a systematic review. BMC Pregnancy Childbirth. 2017;17(1):86.

53. Denno DM, Hoopes AJ, Chandra-Mouli V. Effective strategies to provide adolescent sexual and reproductive health services and to increase demand and community support. J Adolesc Health. 2015;56(1):22-41.

54. Ambresin A-E, Bennett K, Patton GC, Sanci LA, Sawyer SM. Assessment of youth-friendly health care: a systematic review of indicators drawn from young people's perspectives. J Adolesc Health. 2013;52(6):670-81.

55. OPTIONS Consortium. Provider training package: effective oral PrEP delivery for adolescent girls and young women. Washington (DC): OPTIONS Consortium; 2019. https://www.prepwatch.org/resource/effective-delivery-oral-prep-agyw/. Accessed 21 Sep 2020.

56. Cooper LA, Roter DL, Carson KA, Beach MC, Sabin JA, Greenwald AG, et al. The associations of clinicians' implicit attitudes about race with medical visit communication and patient ratings of interpersonal care. Am J Public Health. 2012;102(5):979-87.

57. Hartmann M, Lanham M, Palanee-Phillips T, et al. Generating CHARISMA: development of an intervention to help women build agency and safety in their relationships while using PrEP for HIV prevention. AIDS Educ Prev. 2019;31(5):433-51.

58. O’Malley G, Barnabee G, Mugwanya K. Scaling-up PrEP delivery in sub-Saharan Africa: what can we learn from the scale-up of ART? Curr HIV-AIDS Rep. 2019;16(2):141-50.

59. Sharpe JD, Kamara MT. A systematic evaluation of mobile apps to improve the uptake of and adherence to HIV pre-exposure prophylaxis. Sex Health. 2018;15(6):587-94.

60. Hosek S, Celum C, Wilson CM, Kapogiannis B, Delany-Moretlwe S, Bekker L-G. Prevention HIV among adolescents with oral PrEP: observations and challenges in the United States and South Africa. J Int AIDS Soc. 2016;19(Suppl 6):21107.

61. Moskowitz DA, Macapagal K, Mongrella M, Pérez-Cardona L, Newcomb ME, Mustanski B. What if my dad finds out!?: Assessing adolescent men who have sex with men's perceptions about parents as barriers to PrEP uptake. AIDS Behav. 2020;24(9):2703-19. 
62. Nyblade L, Stockton MA, Giger K, Bond V, Ekstrand ML, Mc Lean R, et al. Stigma in health facilities: why it matters and how we can change it. BMC Med. 2019;17(1):1-15.

63. OPTIONS Consortium. OPTIONS HIV prevention ambassador training package Durham (NC): FHI 360; 2019. Available from: https://www.prepwatch.org/resource/ambassador-training-package/.

64. Velloza J, Khoza N, Scorgie F, et al. The influence of HIV-related stigma on PrEP disclosure and adherence among adolescent girls and young women in HPTN 082: a qualitative study. J Int AIDS Soc. 2020;23(3):25463.

65. Golub SA. PrEP stigma: implicit and explicit drivers of disparity. Curr HIV-AIDS Rep. 2018;15(2):19097.

66. Stankevitz K, Schwartz K, Hoke T, Li Y, Lanham M, Mahaka I, et al. Reaching at-risk women for PrEP delivery: what can we learn from clinical trials in sub-Saharan Africa? PloS One. 2019;14(6):e0218556.

67. Amico KR, Ramirez C, Caplan MR, Montgomery BE, Stewart J, Hodder S, et al. Perspectives of US women participating in a candidate PrEP study: adherence, acceptability and future use intentions. J Intl AIDS Soc. 2019;22(3):e25247.

68. OPTIONS Consortium. Provider training package: effective delivery of oral PrEP for adolescent girls and young women. Washington (DC): OPTIONS Consortium. 2019. https://www.prepwatch.org/resource/effective-delivery-oral-prep-agyw/. Accessed 21 Sep 2020.

\section{Supplementary Files}

This is a list of supplementary files associated with this preprint. Click to download.

- AdditionalFile1.pdf

- AdditionalFile2.doc

- COREQChecklist.pdf 